\title{
Studies on the effect of surface properties on the biocompatibility of polyurethane membranes
}

\author{
Dong-Tsamn Lin ${ }^{\mathrm{a}}$, Tai-Horng Young ${ }^{\mathrm{b}, *}$, Yu Fang ${ }^{\mathrm{b}}$ \\ ${ }^{a}$ Department of Laboratory Medicine, College of Medicine, National Taiwan University, Taipei 10016, Taiwan, ROC \\ ${ }^{\mathrm{b}}$ Institute of Biomedical Engineering, College of Medicine, National Taiwan University, Taipei 10016, Taiwan, ROC
}

Received 1 December 1999; accepted 28 September 2000

\begin{abstract}
To study the effect of surface properties on the biocompatibility of biomaterials based on the same material, polyurethane membranes with different surface properties were prepared. Myoblast culture and interleukin-1 (IL-1) generation in an air pouch model and in vitro monocyte culture were used to examine biocompatibility of different polyurethane membranes. Polyurethane membranes were found to exhibit significant differences depending on their surface properties prepared by different fabrication processes. When myoblasts were cultured on polyurethane surfaces, the smooth and hydrophobic membrane (F1), prepared by the solvent evaporation process, showed the greatest inhibition of myoblast adhesion compared with other porous and hydrophilic membranes (F2, F3 and F4), prepared by immersing the polymer solution into a precipitation bath. In contrast, IL-1 generation by monocytes/macrophages on the membrane F1 was more severe than those on the porous and hydrophilic membranes. Based on our results, the interaction of biomaterials with various cells is discussed. C) 2001 Elsevier Science Ltd. All rights reserved.
\end{abstract}

Keywords: Polyurethane membranes; Surface properties; Myoblasts; Monocytes/macrophages

\section{Introduction}

The recent progress in biomaterials has enhanced the advancement in orthopedic surgery. The interactions of bone cells with biomaterials is the subject of intensive studies because materials can help the regeneration of bone tissue or lead to the release of powerful osteolytic factors [1-5]. However, few references described the interaction of biomaterials with the skeletal muscles [6-8]. After the implantation of biomaterials, the skeletal muscles around the biomaterials may be adversely affected by the cell-material interaction. It is impossible for bone defect to heal when the muscle tissue around bone defect is seriously hurt. On the other hand, when a foreign substance comes in contact with the organism, the system initiates its host-defense mechanisms involving in the inflammatory response. Macrophage is the major inflammatory cell type found on the surface of biomaterials and is responsible for the foreign-body reac-

\footnotetext{
* Corresponding author. Tel.: + 886-2-23970800 X1455; fax: + 8862-2394-0049.

E-mail address: thyoung@ha.mc.ntu.edu.tw (T.-H. Young).
}

tion. Cytokines, such as interleukin-1 (IL-1), released by the stimulated macrophages can regulate the growth of fibroblasts and induce other cells, such as T lymphocytes to proliferate, synthesize proteins and secrete factors further activating the macrophages to result in the so-called whole body inflammatory response [9-14]. Since cytokines contribute to the inflammatory response and may augment an inflammatory response, numerous investigations have been devoted to IL-1 production by stimulated monocytes adhered to biomaterials [9-14].

Considering these reasons, myoblast culture and IL-1 generation in an air pouch model [14] and in vitro monocyte culture were used to examine biocompatibility of biomaterials. Polyurethane was chosen as the substrate for cell culture, due to their good physical and mechanical properties, along with fairly good biocompatibility and antithrombogenicity characteristics [15]. The polyurethane was used in the form of a membrane but with different surface properties prepared by different fabrication processes. Therefore, the effect of surface properties on the biocompatibility based on the same material was examined by using myoblasts and macrophages in this study. 


\section{Materials and methods}

\subsection{Membrane preparation and characterization}

Polyurethanes used in this study were the commercial Pellethane 2103-80AE purchased from Dow Chemical Company. Membranes were prepared by the phase inversion method [16]. Pellethane elastomers were dissolved in chemical reagent grade dimethyl formamide (DMF) to obtain a $15 \%$ polyurethane solution. Polyurethane solution was spread on a glass plate in a uniform thickness of $275 \mu \mathrm{m}$ by an autocoater (KCC303, RK Print-Coat Instruments, UK) at $25^{\circ} \mathrm{C}$ to prepare membranes. In this work, four polyurethane membranes were prepared by changing the phase inversion process. Membrane $\mathrm{F} 1$ was prepared by evaporating DMF in a vacuum oven at $60^{\circ} \mathrm{C}$ for 2 days. Membranes F2, F3 and F4 were prepared by immersing the casting solution immediately (without evaporation) into a precipitation bath for 1 day. The precipitation medium was water, isopropanol and acetone for membranes F2, F3 and F4, respectively. De-ionized and ultrafiltrated water was used in our experiments. Isopropanol and acetone were of chemical reagent grade and were used without further purification. After the evaporation and precipitation were completed, the membranes were removed from the glass plate and kept in water at $25^{\circ} \mathrm{C}$ for $24 \mathrm{~h}$.

The morphology of the membrane was examined using a scanning electron microscope (SEM). The freeze-dried samples were sputtered with gold and palladium in a vacuum using a Hitachi S-800 microscope at $20 \mathrm{kV}$.

Air-water and octane-water contact angles were measured on polyurethane membranes at $25^{\circ} \mathrm{C}$ using a reverse air-bubble apparatus (CA-D, Kyowa Scientific Co.) to obtain surface energetic information. The polar and dispersive components of the surface-free energy, and solid-water interfacial energy were calculated using the harmonic mean approximation [17].

$\mathrm{X}$-ray photoelectron spectroscopy (XPS) analysis of the polyurethane membranes was performed using an ESCA PHI 1600 photoelectron spectrophotometer (Physical Electronics; USA) with a magnesium anode $(\mathrm{MgK}=1253.6 \mathrm{eV})$. Polyurethane membranes were irradiated with photons from a soft X-ray source with a well-defined energy. The angle between the sample and electron detector was $45^{\circ}$. The survey scan was from 0 to $1000 \mathrm{eV}$, to find the atoms of surface. The relative atomic percentage of each element at the surface was estimated from peak areas using the standard software provided with the instrument.

\subsection{Myoblast culture}

Primary culture of Wistar rat myoblasts was used in this study. Myoblast cells were isolated from male Wistar rats $(250-350 \mathrm{~g})$ by enzymatic digestion technique follow- ing the method of Bischoff [18]. The culture media used was Dulbecco's modified Eagle's medium (MEM) supplemented with $10 \%$ fetal calf serum (Gibco-RBL Life Technologies, Paisley, UK) and $1 \%$ antibiotics (penicillin G sodium $100 \mathrm{U} / \mathrm{ml}$-streptomycin $100 \mathrm{U} / \mathrm{ml}$, Gibco-RBL Life Technologies, Paisley, UK).

Circular samples ( $1.5 \mathrm{~cm}$ in diameter) were cut from the polyurethane membranes, rinsed extensively with phosphate-buffered saline (PBS) and then placed in the 24well tissue culture plate (Corning, New York, USA). After overnight sterilization under ultraviolet light cell suspensions were added to the well at a density of $1 \times 10^{5}$ cells/well in DMEM medium. Cell cultures were maintained in a humidified atmosphere with $5 \% \mathrm{CO}_{2}$ at $37^{\circ} \mathrm{C}$.

For morphological observation, the cells adhering to the membrane were washed with PBS after $4 \mathrm{~h}$ incubation and then fixed with $2.5 \%$ glutaraldehyde in PBS at $4^{\circ} \mathrm{C}$ for $1 \mathrm{~h}$. Subsequently, the cells were dehydrated by graded ethanol changes and then critical point dried. The dried samples were then gold sputtered in vacuum and examined by SEM. On the other hand, the number of attached myoblasts $24 \mathrm{~h}$ after cell seeding was measured. The membrane surface was washed with PBS twice to remove cell debris present in the medium. Adhering cells were detached with trypsin, stained with trypan blue to ensure the cell viability, and counted using a Neubauer counting chamber under an inverted microscope [6]. For each experimental value, four independent experiments were carried out.

\subsection{IL-1 production in an air pouch model}

IL-1 is produced in both soluble and membrane-associated forms [19]. Membrane-associated IL-1 (mIL-1) is adherent to the leukocyte membrane or the surface of biomaterials that depends on the morphology and composition of material [13]. In this work, air pouches were used as an in vivo culture system to measure the mIL-1 produced on the surface of polyurethane membranes according to the method of Edwards et al. [20]. Wistar rats $(250-350 \mathrm{~g})$ were anesthetized using a mixture of combelen (Bayer, Germany) and ketamine (Sintong, Taiwan) $(v / v=1 / 1)$. A subcutaneous injection of $20 \mathrm{ml}$ of prefiltered air was made on the dorsal area to form an air pouch. This air pouch was kept inflated by reinjecting with $10 \mathrm{~cm}^{3}$ of air every 3 days. On day 6 , the rats were again anesthetized and prepared for surgery by shaving the air pouch and then scrubbing with alcohol. Four different polyurethane membranes were implanted on the inner surface of the air pouch. At 4, 8, 12, 24 and $48 \mathrm{~h}$, the rats were sacrificed and the implants were removed and immediately fixed with $1 \%$ paraformaldehyde for $15 \mathrm{~min}$ at $37^{\circ} \mathrm{C}$ following the method of Kurt-Jones et al. [19]. There were three animals for each time point. After fixation, the membranes were placed in 24-well tissue 
culture polystyrene plates and cultured in a humidified atmosphere of $95 \%$ air $/ 5 \% \mathrm{CO}_{2}$ at $37^{\circ} \mathrm{C}$. The culture media used were RPMI-1640 medium supplemented with $10 \%$ fetal bovine serum (Gibco-RBL Life Technologies, Paisley, UK) and $1 \%$ antibiotics. After $24 \mathrm{~h}$ of incubation, supernatant was harvested and IL-1 concentration was measured by ELISA procedure.

\subsection{In vitro test of $I L-1$}

Venous blood was obtained from normal adult volunteers in accordance with the guidelines of National Taiwan University Hospital. Human peripheral blood mononuclear cells were isolated by Percoll density gradient sedimentation procedure $[11,21]$. The mononuclear cells were harvested and washed with cold PBS without $\mathrm{Ca}^{2+}$ and $\mathrm{Mg}^{2+}$ (Gibco, Grand Island, NY) to minimize aggregation and resuspended in RPMI-1640 medium. The monocyte purity was greater than $85 \%$. The mononuclear cells were seeded at $1 \times 10^{5}$ cells/well without and with lipopolysaccharide (LPS, $5 \mu \mathrm{g} / \mathrm{ml}$ ) and cultured for $24 \mathrm{~h}$. Supernatant was harvested and IL-1 concentration was measured by ELISA procedure. All experiments were repeated four times.

\subsection{Data analysis}

Data were presented as the mean \pm standard deviation. Statistical significance was calculated using one way analysis of variance (ANOVA) followed by Student's $t$-test $(p<0.05$ was considered significant).

\section{Results}

\subsection{Membrane characterization}

Macroscopically, membrane F1 was transparent and membranes F2, F3 and F4 appeared opaque. The microscopic analysis shows that the membrane structures varied with different preparation procedures. The direct evaporation of the casting polymer solution produced membrane F1 with a fairly dense and smooth structure, as indicated in Fig. 1. In contrast, as the casting polymer solution was precipitated in water, the membrane F2 consisted of a surface with superficial pores supported by a porous sublayer that was occupied by macrovoids, as indicated in Fig. 2. Dissimilar to membrane F2, the surface pores of membrane F3, precipitated in isopropanol, were open to the interior and the sublayer structure changed one from with macrovoids to that with sponge-like pores, as indicated in Fig. 3. The structure of membrane $\mathrm{F} 4$, which was prepared by precipitation in acetone, is shown in Fig. 4. Both the top surface and cross section were very porous. Especially, pores in the cross section appeared to be enclosed in irregular brick-like aggregates. Altogether, membranes F2, F3 and F4, prepared by immersing the casting solution into a precipitation bath, had porous surfaces. The diameters of surface pores were estimated to be approximate 1,5 and $5 \mu \mathrm{m}$ for membranes F2, F3 and F4, respectively.

Contact angle data and calculated surface energetic results for the prepared membranes are shown in Table 1. The solid-water interfacial energy for the membrane F1 was very high, while those for membranes F2, F3 and F4 were gradually tending to zero. This indicates that the
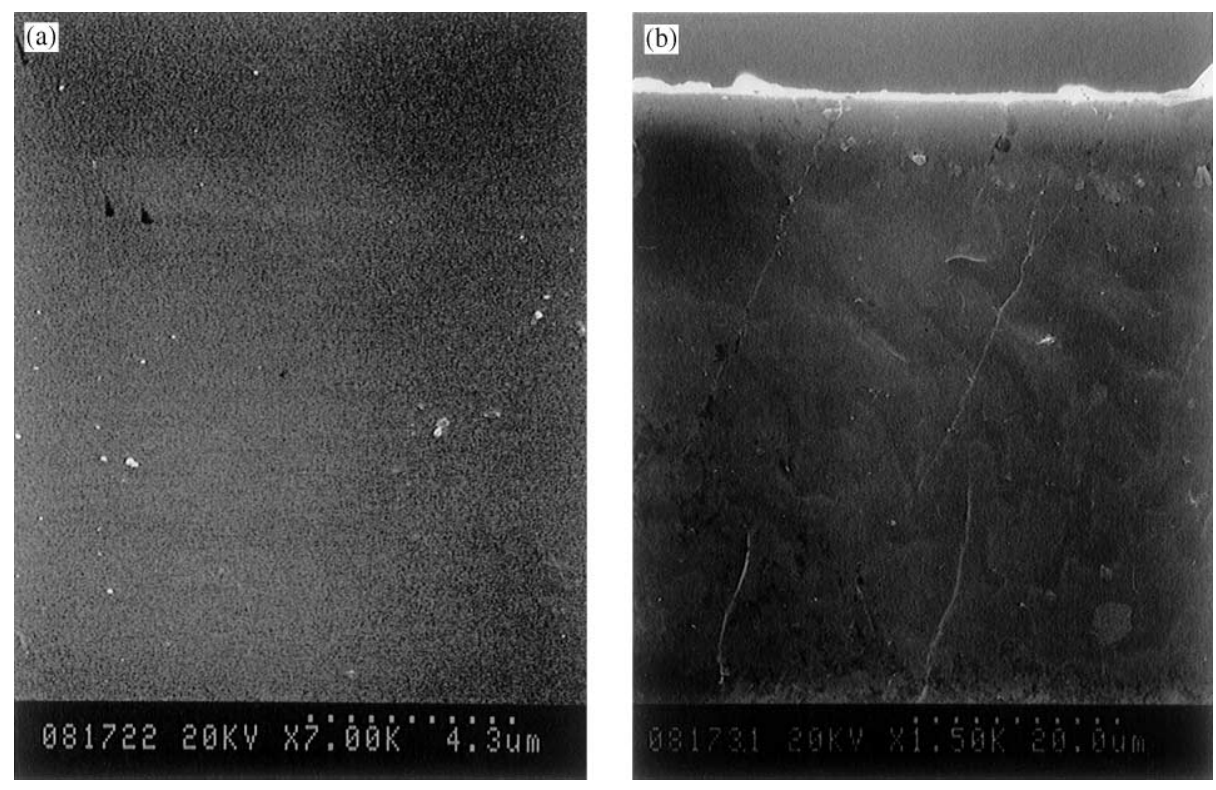

Fig. 1. SEM photomicrographs of membrane F1. (a) Top surface; (b) cross section. 

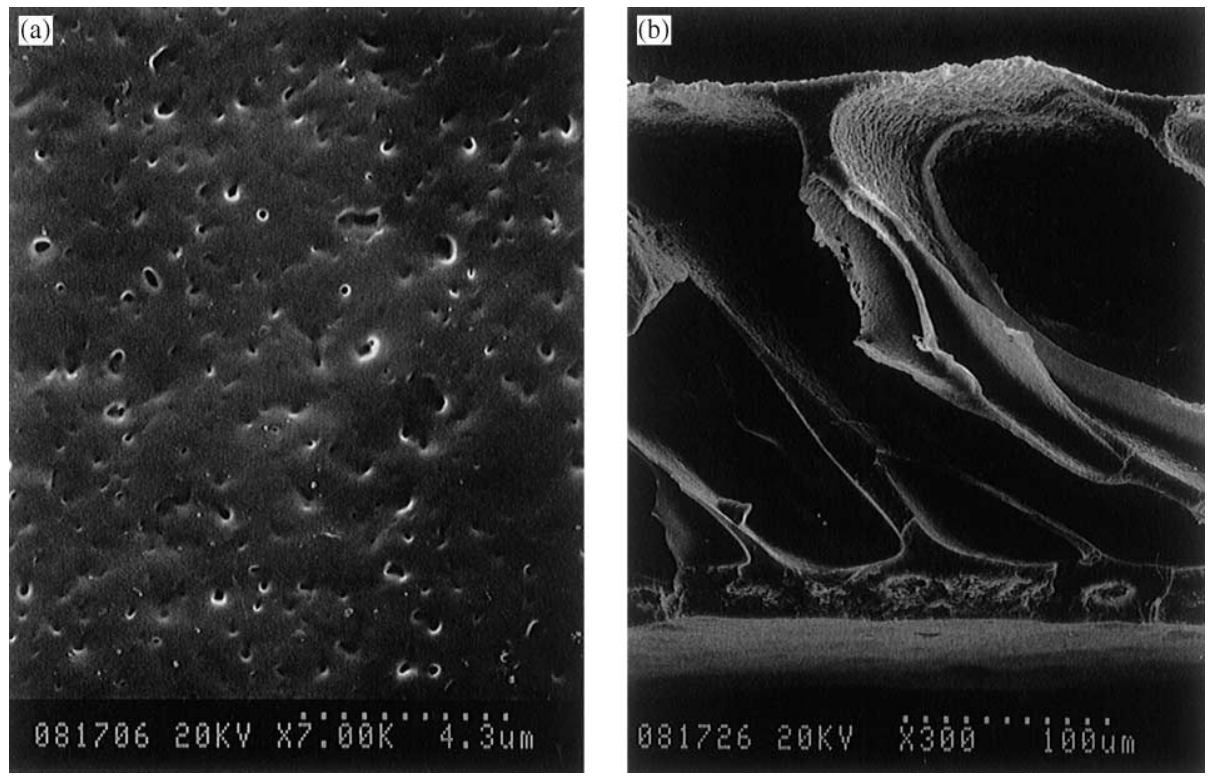

Fig. 2. SEM photomicrographs of membrane F2. (a) Top surface; (b) cross section.
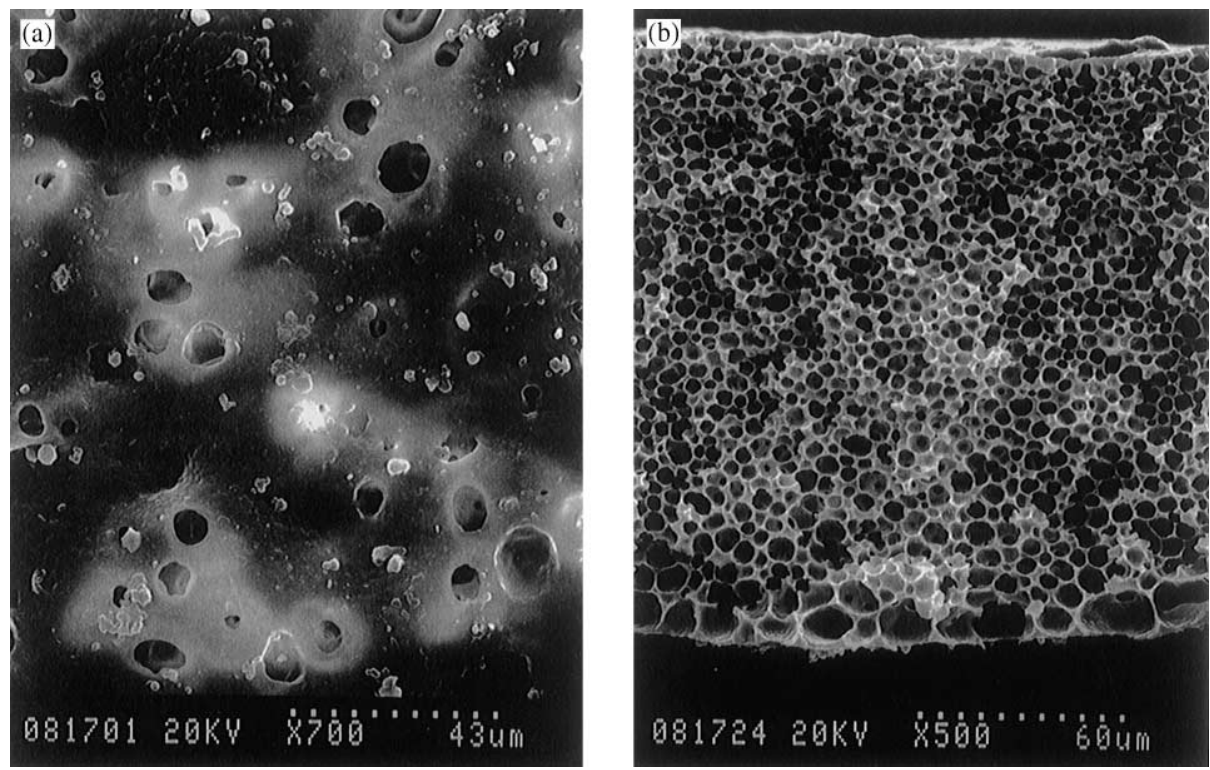

Fig. 3. SEM photomicrographs of membrane F3. (a) Top surface; (b) cross section.

membranes prepared by the precipitation process were more hydrophilic compared with membranes prepared by the solvent evaporation process. Moreover, membrane F4 was relatively the most hydrophilic membrane in our study, presumably due to the hydrophilic segments selectively being present at the surface in the course of preparation of the membrane. Likewise, the least hydrophilic surface of membrane F1 was attributed to hydrophilic segments embedded into the deeper region at the time of evaporation of the solvent from the cast polymer solution. Therefore, polymer chain rearrangement with different preparation procedures has a great influence on the membrane surface chemistry, which can be further examined by XPS (shown below).

Table 2 shows the surface composition analysis for the prepared polyurethane membranes. Although the low concentration of the hard segment phase in the surface limited accurate quantification of the nitrogen content, Table 2 shows the surface carbon content was in the order of $\mathrm{F} 1>\mathrm{F} 2>\mathrm{F} 3>\mathrm{F} 4$ and the surface oxygen content was in the order of F4 $>$ F3 $>$ F2 $>$ F1. Thus, relatively more hydrophilic membranes prepared by the precipitation process appeared to be primarily caused by a loss in the surface $\mathrm{C} / \mathrm{O}$ atomic ratio. 

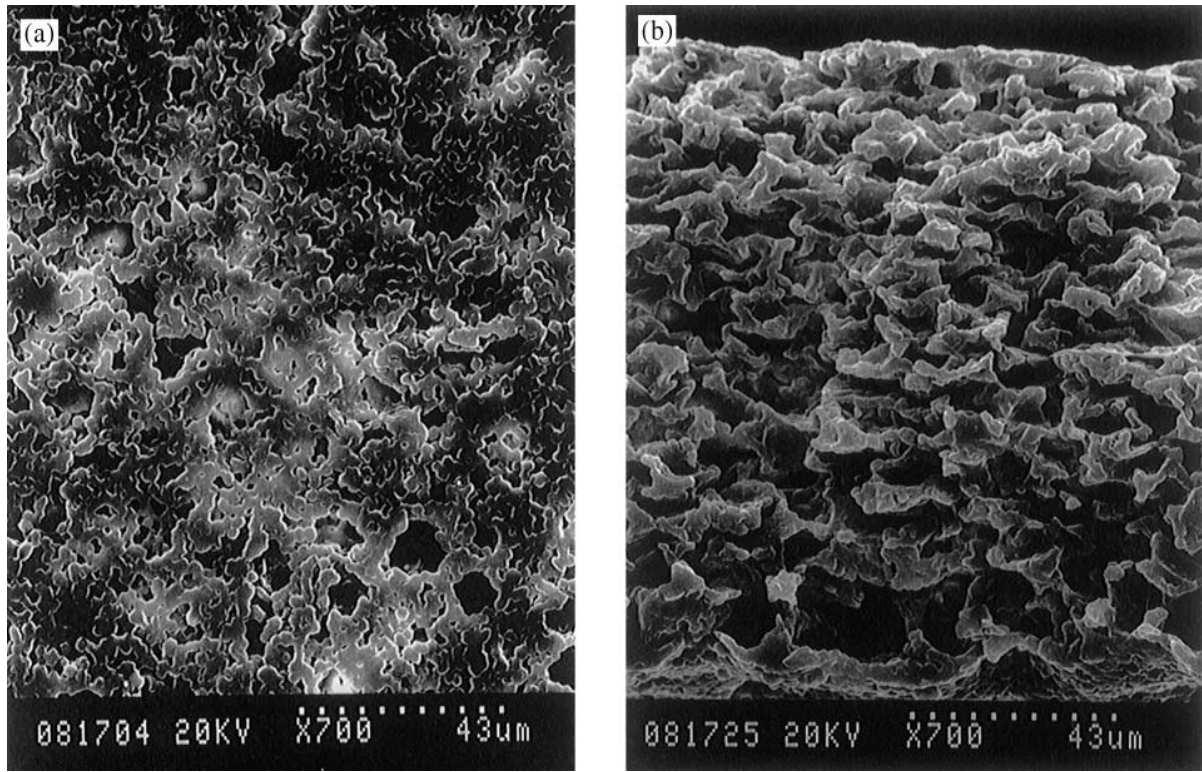

Fig. 4. SEM photomicrographs of membrane F4. (a) Top surface; (b) cross section.

Table 1

Contact angle and surface energetic results for different polyurethane membranes ${ }^{\mathrm{a}}$

\begin{tabular}{|c|c|c|c|c|}
\hline Membrane & $\begin{array}{l}\text { Air-water contact } \\
\text { angle (deg) }\end{array}$ & $\begin{array}{l}\text { Octane-water contact } \\
\text { angle (deg) }\end{array}$ & $\begin{array}{l}\text { Solid-water interfacial } \\
\text { energy }\left(\mathrm{erg} / \mathrm{cm}^{2}\right)\end{array}$ & Polar/dispersive ratio \\
\hline $\mathrm{F} 1$ & $54.1 \pm 0.6$ & $40.6 \pm 0.8$ & 3.90 & 1.30 \\
\hline $\mathrm{F} 2$ & $36.4 \pm 1.8$ & $24.7 \pm 1.9$ & 1.14 & 1.65 \\
\hline F3 & $27.6 \pm 1.6$ & $17.8 \pm 1.7$ & 0.44 & 1.87 \\
\hline $\mathrm{F} 4$ & $13.6 \pm 0.8$ & $17.6 \pm 1.1$ & 0.10 & 2.49 \\
\hline
\end{tabular}

${ }^{\mathrm{a}}$ Values are means of 10 measurements \pm standard deviation.

Table 2

XPS results for various polyurethane membranes: surface composition $(\mathrm{at} \%)$

\begin{tabular}{llll}
\hline Membrane & $\mathrm{C}_{1 \mathrm{~s}}$ & $\mathrm{O}_{1 \mathrm{~s}}$ & $\mathrm{~N}_{1 \mathrm{~S}}$ \\
\hline F1 & 75.9 & 20.6 & 3.5 \\
F2 & 75.2 & 22.1 & 2.7 \\
F3 & 72.2 & 23.0 & 4.8 \\
F4 & 69.6 & 24.8 & 5.6 \\
\hline
\end{tabular}

\subsection{Myoblast culture}

After cells contact a biomaterial, cells will alter their cell membrane to stabilize the cell-biomaterial interface [22]. Hence, cellular morphology of a biomaterial can be used to evaluate the biocompatibility of a biomaterial. In this work, SEM photographs of Wistar rat myoblasts on the prepared membranes after culturing for $4 \mathrm{~h}$ were studied to evaluate observable differences among the morphologies of myoblast cells. The whole process of cell adhesion and spreading consists of cell attachment, filopodial growth, cytoplasmic wedding, flattening of the cell mass and ruffling of peripheral cytoplasm progressing, all of which progress in a sequential fashion [23]. The adherent cells on the membrane F1 were not spread at all and remained spherical in appearance (Fig. 5(a)). This indicates that myoblast cells cultured on this smooth and hydrophobic surface had a poor morphology without the growth of filopodia. Fig. 5(d) shows the morphology of myoblast cells cultured on the surface of membrane F4. Myoblasts cells on the most porous and the most hydrophilic surface in this study were also spherical in appearance but the cells' surfaces appeared rough, with foldings and filopodia began to extend radially. Fig. 5(b) and (c) show the morphology of myoblast cells attached onto the surfaces of membranes F2 and F3, respectively. SEM examination revealed that the myoblast cells with flattened morphology, indicating these two porous membranes with moderate surface hydrophilicity, do not provide a stimulus to myoblast cells and are consistent with the situation expected for biocompatible biomaterials. The result was supported by the 

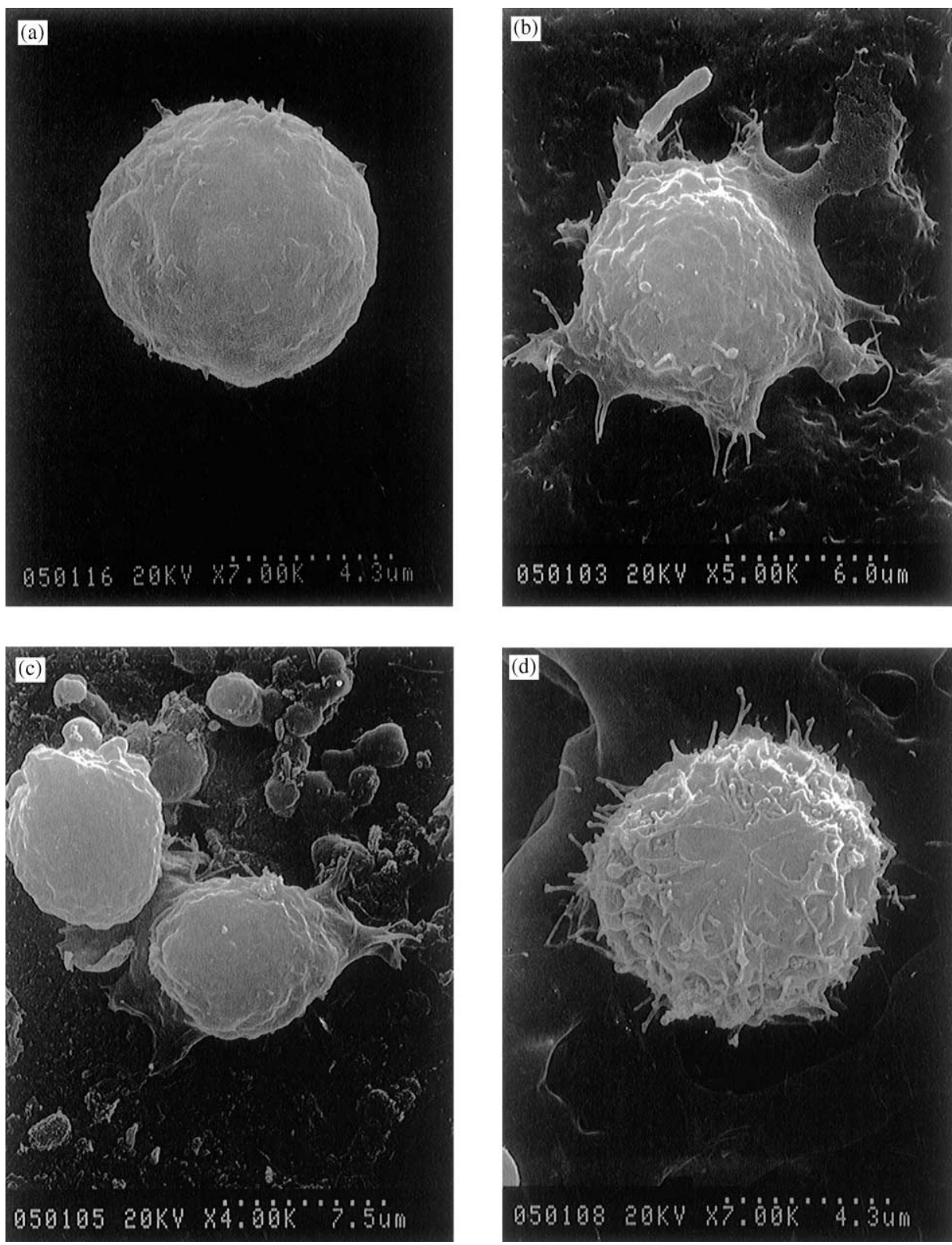

Fig. 5. SEM photomicrographs of myoblasts adhered on various polyurethane membranes after 4 h culture. (a) Membrane F1; (b) membrane F2; (c) membrane F3 (d) membrane F4.

work of others. Wachem et al. [24] demonstrated that human endothelial cells adhere and spread preferably on moderately wettable polymers with a water contact angle of $35^{\circ}$. Lee and Lee [25] found the maximum adhesion of the Chinese hamster ovary cells appeared at around a water contact angle of $55^{\circ}$.

Fig. 6 shows the amount of myoblast cells attached to the various surfaces of prepared membranes after culturing for $24 \mathrm{~h}$. The amount of cell adhesion was expressed as a percentage of the number of cells adhering to the control (tissue culture polystyrene). Membrane surface structure was found to influence cell adhesion greatly. There was a significance difference $(p<0.05)$ in the cell adhesion on membranes F3 and F4 compared to the cell adhesion on membrane F1. However, membrane F2 only had a slight higher cell adhesion than membrane F1. Summarizing from membrane surface structure and the amount of cell adhesion, porous membranes had higher cell adhesion compared with the smooth membrane and the amount of myoblast cells present on the membrane increased with increasing the pore size on the membrane surface. It is reasonable to hypothesize that the amount of cell adhesion would increase as the area of its contact with the membrane surface increases; however, it cannot promise that the process of cell adhesion is in a more progressive stage. For example, membrane F2 did not 
have the highest amount of cell adhesion, but it showed myoblast cells forming a flattened morphology over the membrane surface (Fig. 5(b)), suggesting myoblasts tended to adhere firmly on the membrane F2 surface. Considering the effect of membrane surface hydrophilicity and structure on the culture of myoblast cells, the membrane F1 with smooth and hydrophobic surface appeared to be the most unsuitable for the myoblast culture.

\subsection{IL-1 production}

The rodent air pouch was first reported by Edwards et al. [20]. The air pouch could be opened and then materials could be placed inside to study macrophages adherent to the materials by using a variety of methods. Kurt-Jones et al. showed that macrophages became activated to produce mIL-1 adhering to the polystyrene

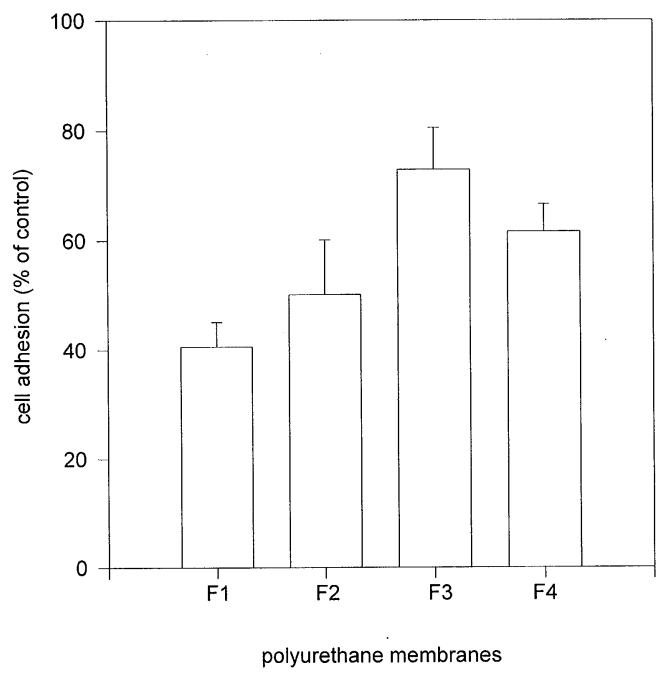

Fig. 6. The number of myoblasts as a percentage of the control on different polyurethane membranes after $24 \mathrm{~h}$ culture $(n=4)$. substrate [19]. In this study, this model was used to compare the quantities of mIL-1 localized on the different polyurethane membranes. As indicated in Fig. 7, membrane $\mathrm{F} 1$ induced a rapid increase in $\mathrm{mIL}-1$ production within the first $10 \mathrm{~h}$ of incubation. The mIL-1 production which was maximal at $4 \mathrm{~h}$, subsequently decreased to very low levels by $12 \mathrm{~h}$. In contrast, a rapid increase in $\mathrm{mIL}-1$ production was not observed for membranes F2, F3 and F4 and the maximum mIL-1 production shifted to a longer time 8,12 and $24 \mathrm{~h}$ for membranes F2, F3 and F4, respectively. Although the maximum mIL-1 generation did not appear at the same time, the mIL-1 generation increased to the maximum within $48 \mathrm{~h}$ for all membranes.

The air pouch model approximates in vivo conditions and can be used to evaluate the biocompatibility of prepared membranes. When the data were evaluated based on the maximum mIL-1 production dependence, Fig. 7 shows the level of mIL-1 production on the membrane F1 was significantly higher compared with other membranes (F2, F3 and F4) $(p<0.05)$. This means that membranes F2, F3 and F4 were more bioinert than membrane F1. It is reasonable to regard the mIL-1 production is proportional to the number of macrophages attached to polyurethane membranes. However, this model cannot control the number of cells adhering to the membrane. Therefore, a biomaterial might induce macrophages to release higher amounts of IL-1, but it is not certain that more macrophages attach to this biomaterial. In fact, culturing myoblasts in vitro, the amount of cell adhesion on different membranes was also different significantly; see Fig. 6 . This gives us a basis for the interpretation of cell behavior on the biomaterials presented in the discussion section.

Fig. 8 shows the amount of IL-1 released by human monocytes in vitro into the medium, including the exposures of prepared membranes with and without LPS. After $24 \mathrm{~h}$ incubation, the trend of IL-1 production in the

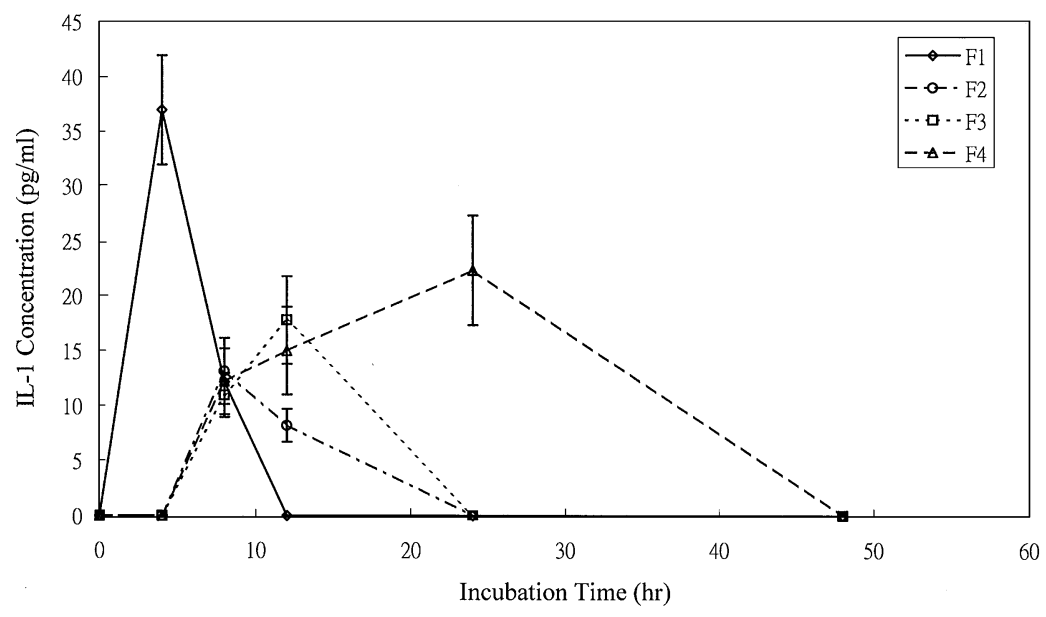

Fig. 7. Generation of mIL-1 on the surface of polyurethane membranes by using the rodent air pouch model at different implantation times $(n=3)$. 


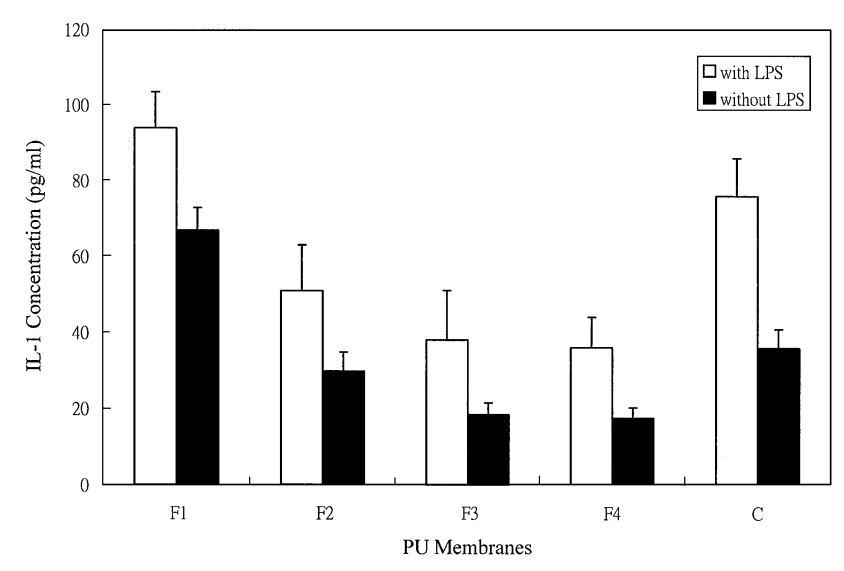

Fig. 8. Generation of IL-1 of in vitro exposure of polyurethane membranes to the human monocytes with and without LPS $(n=4)$.

monocyte culture medium for various membranes with and without LPS was similar and IL-1 generated in the absence of LPS showed significantly lower levels than that in the presence of LPS for the same sample $(p<0.05)$. When the data were evaluated based on the presence of LPS, the level of IL-1 production for membranes F2, F3 and F4 was similar but they exhibited significant inhibitory effect on IL-1 release compared with control ( $p<0.05)$. By contrast, there was no significant difference in the IL-1 production between membrane F1 and control. This result reinforces the in vivo studies in the air pouch model.

\section{Discussion}

To examine the effect of surface properties on the biocompatibility of biomaterials, we have prepared polyurethane membranes by different fabrication processes to exhibit different surface properties. We found that the membrane surface structure and hydrophilicity could be changed greatly. It is desirable to characterize the effect of such differences on the biocompatibility tests for the specific requirement of biomaterials. Although the interaction of biomaterials with various cell types has been studied from many viewpoints, as described in the introduction we used myoblasts and macrophages/monocytes to evaluate the biocompatibility of polyurethane membranes.

The experiments in this study indicate that the trend of pore size on the membrane surface was consistent with the result of myoblast adhesion. When polyurethane membranes were subjected to myoblasts in vitro, the membrane F1 showed less cell adhesion on the smooth and dense surfaces than on the rough and porous surfaces. In contrast, the myoblast morphology appeared to be determined by membrane surface hydrophilicity. Membranes F2 and F3 with moderate surface hy- drophilicity offered more favorable surfaces for myoblast cells forming a more flattened morphology over the membrane surfaces. In addition, membrane $\mathrm{F} 1$ was the most stimulating to IL-1 production from macrophages in the air pouch model and in vitro culture. But porous membranes F2, F3 and F4 regardless of hydrophilicity did not stimulate macrophages to produce a high level of IL-1. Dissimilar to the result of myoblast culture, membrane surface hydrophilicity did not influence the release of IL-1 from macrophages. This suggests macrophages appeared less activated on porous surfaces than those did on dense surfaces. Therefore, polyurethane membrane with proper surface structure and hydrophilicity may be beneficial as an implant material, since it can exhibit inhibitory effect on IL-1 release by macrophages but does not inhibit adhesion of myoblasts.

Results obtained in the present study indicate that polyurethane membranes prepared by different fabrication processes exhibit different biocompatible properties. One question about the membrane F1, however, remains to be solved. Both in the air pouch model and in vitro monocyte culture, IL-1 production demonstrated macrophages had higher activity on the membrane F1. Although myoblasts and macrophages are different cells with different adhesion characteristics, based on the result of myoblast culture, we assume the number of macrophages adhering on the membrane also increases as the pore size on the membrane surface is increased. In fact, Young et al. have found porous surfaces enhanced monocyte adhesion and nonporous surfaces showed the inhibition of monocyte adhesion in different polymers [26]. Further, we assume the contribution of nonadherent macrophages in releasing IL-1 is negligible. Therefore, we propose that even though a biomaterial surface is least likely to attach macrophages, it may activate macrophages to elicit a greater amount of IL-1.

The interaction between the host and the biomaterials is a dynamic process. In the beginning macrophages adhere to the surface of the foreign body. Subsequently, macrophages are activated to produce cytokines. Therefore, the first step in a successful host-foreign body interaction depends on an adequate surface for host cells to adhere but not to activate to release cytokines. There can be two possible mechanisms for a high level of IL-1 released by fewer macrophages on the membrane F1. The first possible mechanism is that the membrane F1 directly stimulate fewer adhering macrophages to release a high level IL-1. Thus, the interaction of macrophages with the biomaterial surface consists of two independent stages: cell adhesion and cell activation [26]. In other words, the generation of cytokine by monocytes is not proportional to the number of cells adherent to the surface. The second possibility is that a high level IL-1 released by macrophages on membrane F1 is indirectly stimulated by membrane $\mathrm{F} 1$, which is probably preceded by a progressive apoptosis in macrophages. When 
macrophages contact membrane F1, less cell adhering onto the membrane surface lead to more cell death in the medium. Therefore, membrane $\mathrm{F} 1$ gives rise to high concentration of proteolytic enzymes and excitotoxic amino acids derived from dead cells to stimulate adhering macrophages to release cytokines. Also, the macrophage behavior on a biomaterial probably combines aspects of both possibilities. In conclusion, membrane F1 is not suitable for cell culture, but the detailed mechanism needs further investigations.

\section{Acknowledgements}

The authors thank the National Science Council of the Republic of China for their financial support.

\section{References}

[1] Morrison C, Macnair R, MacDonald C, Wykman A, Goldie I, Grant MH. In vitro biocompatibility testing of polymers for orthopaedic implants using cultured fibroblasts and osteoblasts. Biomaterials 1995;16:987-92.

[2] Saad B, Matter S, Ciardelli G, Uhlschmid GK, Welti M, Neuenschwander P, Suter UW. Interactions of osteoblasts and macrophages with biodegradable and highly porous polyesterurethane foam and its degradable products. J Biomed Mater Res 1996; 32:355-66

[3] Kieswetter K, Schwartz Z, Hummert TW, Cocharn DL, Simpson J, Dean DD, Boyan BD. Surface roughness modulates the local production of growth factors and cytokines by osteoblast-like MG-63 cells. J Biomed Mater Res 1996;32:55-63.

[4] Koh ET, Torabinejad M, Ford TRP, Brady K, McDonald F. Mineral trioxide aggregate stimulates a biological response in human osteoblasts. J Biomed Mater Res 1997;37:432-9.

[5] Sohrabi A, Holland C, Kue R, Nagle D, Hungerford DS, Frondoza CG. Proinflammatory cytokine expression of IL- $1 \beta$ and TNF- $\alpha$ by human osteoblast-like MG- 63 cells upon exposure to silicon nitride in vitro. J Biomed Mater Res 2000;50:43-9.

[6] Derker C, Greggs R, Duggan K, Stubbs J, Horwitz A. Adhesive multiplicity in the interaction of embroyonic fibroblasts and myoblasts with extracellular matrices. Cell Biol 1984;99:1398-404.

[7] Sun JS, Tsuang YH, Yao CH, Liu HC, Lin FH, Hang YS. Effects of calcium phosphate bioceramics on skeletal muscle cells. J Biomed Mater Res 1997;34:227-34

[8] Young TH, Yao CH, Sun JS, Lai CP, Chen LW. The effect of morphology variety of EVAL membranes on the behavior of myoblasts in vitro. Biomaterials 1998;19:717-24.
[9] Dinarello CA. An update on human interleukin-1: from molecular biology to clinical relevance. J Clin Immunol 1985;5:287-97.

[10] Miller KM, Anderson JM. Human monocyte/macrophage activation and interleukin 1 generation by biomedical polymers. J Biomed Mater Res 1988;22:713-31.

[11] Miller KM, Huskey RA, Bigby LF, Anderson JM. Characterization of biomedical polymer-adherent macrophages: interleukin 1 generation and scanning electron microscopy studies. Biomaterials 1989;10:187-95.

[12] Miller KM, Rose-Caprara V, Anderson JM. Generation of IL-1like activity in response to biomedical polymer implants: a comparison of in vitro and in vivo models. J Biomed Mater Res 1989;23:1007-26.

[13] Cardona MA, Simmons RL, Kaplan SS. TNF and IL-1 generation by human monocytes in response to biomaterials. J Biomed Mater Res 1992;26:851-9.

[14] Krause TJ, Robertson FM, Wasserman AJ, Greco RS. Differential production of interleukin 1 on the surface of biomaterials. Arch Surg 1990;125:1158-60.

[15] Lelah MD, Cooper SI. Polyurethane in medicine. Boca Raton, FL, USA: CRC Press, 1986.

[16] Kesting RE. Synthetic polymeric membranes. New York, NY: Wiley, 1985.

[17] Andrade JD, Ma SM, King RN, Gregonis DE. Contact angles at the solid-water interface. J Colloid Interface Sci 1979;72:488-94

[18] Bischoff R. Enzymatic liberation of myogenic cells from adult rat muscle. Anat Rec 1974;180:645-62.

[19] Kurt-Jones E, Beller D, Mizel S, Unanue E. Identification of a membrane-associated interleukin-1 in macrophages. Proc Natl Acad Sci USA 1985;138:3799-802.

[20] Edwards J, Sedgwick A, Willoughby D. The formation of structure with the features of synovial lining by subcutaneous injection of air: an in vivo tissue culture system. J Pathol 1981;134:147-56.

[21] Pertoft H, Johnsson A, Warmegaro B, Seljeld R. Separation of human monocytes on density gradients of percoll. J Immunol Meth 1980;33:221-9.

[22] Okano ZT, Yamada N, Okuhara M, Sakai H, Sakurai Y. Mechanism of cell detachment from temperature-modulated, hydrophilic-hydrophobic polymer surfaces. Biomaterials 1995; 16:297-303.

[23] Rajaraman R, Rounds DE, Yen SPS, Rembaum A. A scanning electron microscope study of cell adhesion and spreading in vitro. Exp Cell Res 1974;88:327-39.

[24] van Wachem PB, Beugeling T, Feijen J, Bantjes A, Detmers JP, van Aken WG. Interaction of cultured human endothelial cells with polymeric surfaces of different wettabilities. Biomaterials 1985;6:403-8

[25] Lee JH, Lee HB. A wettability gradient as a tool to study protein adsorption and cell adhesion on polymer surface. J Biomater Sci Polym Edn 1993;4:467-81.

[26] Young TH, Lin DT, Chen LY. Human monocyte adhesion and activation on crystalline polymers with different morphology and wettability in vitro. J Biomed Mater Res 2000;50:490-8. 